\title{
FINITE TIMES TO EQUIPARTITION IN THE THERMODYNAMIC LIMIT
}

\author{
by \\ J. De Luca ${ }^{(\mathrm{a})}$, A. J. Lichtenberg ${ }^{(\mathrm{b})}$, S. Ruffo ${ }^{(\mathrm{c})}$ \\ (a) Instituto de Física, Universidade Federal de São Carlos \\ Caixa Postal 676, 13565-905, São Carlos, SP, Brazil \\ (b) Department of Electrical Engineering and Computer Sciences \\ and the Electronics Research Laboratory \\ University of California, Berkeley, CA 94720 \\ (c) Dipartimento di Energetica "S. Stecco" \\ Universitá di Firenze, and INFN, 50139 Firenze, Italy
}

\begin{abstract}
We study the time scale $T$ to equipartition in a $1 D$ lattice of $N$ masses coupled by quartic nonlinear (hard) springs (the Fermi-Pasta-Ulam $\beta$ model). We take the initial energy to be either in a single mode $\gamma$ or in a package of low frequency modes centered at $\gamma$ and of width $\delta \gamma$, with both $\gamma$ and $\delta \gamma$ proportional to $N$. These initial conditions both give, for finite energy densities $E / N$, a scaling in the thermodynamic limit (large $N$ ), of a finite time to equipartition which is inversely proportional to the central mode frequency times a power of the energy density $(E / N)$. A theory of the scaling with $(E / N)$ is presented and compared to the numerical results in the range $0.03 \leq E / N \leq 0.8$.

PACS numbers: 05.45.+b, 63.20.Ry, 63.10.+a
\end{abstract}




\section{INTRODUCTION}

In previous work $[1,2]$, the time scale to achieve equipartition in a nonlinear lattice of masses coupled by hard springs (FPU- $\beta$ model) was studied with the initial energy primarily in a low-frequency mode, of mode number $\gamma$. It was shown in [1], numerically and theoretically, that energy transfer to high frequency modes is exponentially slow in a perturbation (energy) parameter at low energy. The mechanism of a transition to more rapid energy transfer is that for energy above a threshold $E \propto N^{-1}$, interaction of neighboring low frequency modes will lead to a local superperiod beat oscillation, of period $T_{B} \propto N^{2} / \gamma E_{\gamma}$, that is stochastic. At a sufficiently high energy a resonance with high frequency modes leads to a transition to a fast diffusion regime which occurs above a critical energy $E=E_{c}$, independent of $N$. The equipartition time scale for $E>E_{c}$ was studied numerically in $[1,2]$, with the more detailed numerical investigation of the scaling time to equipartition in [2] for $0.2 \leq E / N \leq 1.0$ giving $T \propto T_{B} \sqrt{N}$. The $\sqrt{N}$ was interpreted as a size-dependent correction to the $T_{B}$ time scale. In a heuristic treatment of a more general oscillator chain we indicated a possible explanation for the $\sqrt{N}$ mode filling factor [3]. If we additionally set $\gamma \propto N$ in $T_{B}$, above, then $T_{B}$ only depends on the total energy per mode (energy density). The numerical results in [2] on the other hand, indicated that $T$, having an additional $\sqrt{N}$ dependence, would become infinite with $N$ at constant energy density, and therefore $T$ would not have a finite value in the thermodynamic limit.

In another study [4], the energy was placed in a low frequency package centered on a mode $\gamma \propto N$ and with an extension $\delta \gamma \propto N$. In that work the numerical results indicated that the equipartition time was dependent only on the energy density $E / N$ and therefore remained finite in the thermodynamic limit, for a finite $E / N$. However, the measure of equipartition used in that study was rather insensitive, so that the exact scalings were 
difficult to obtain.

Other work has also discussed the question of time to equipartition for various initial conditions and nonlinearities [5-10]. In an early investigation, at higher energies, the relation between time scales and Lyapunov exponents was investigated [5]. At the higher energies investigated in that study, there was primary mode overlap, for which a weak power law behavior was observed, with a transition to stochasticity governed by a random matrix approximation. The existence of weak chaos at vanishing energy was further explored in Ref. [11]. In other studies of more regular systems, such as the discretized integrable sine Gordon equation [6], and the FPU- $\alpha$ cubic nonlinearity, which is a two term Taylor series approximation to the integrable Toda lattice [8], abrupt transitions were found between power law behaviour and "essentially integrable" behaviour. This class of coupled systems is well worth further study, but has significantly different properties from the more thoroughly studied FPU- $\beta$ system. In another study a $\phi^{4}$ nonlinear chain was introduced to allow the transition between power law and exponentially slow behavior to be studied [9]. The attention was mainly on the exponentially slow time scales in the low energy (small quartic nonlinearity) region, which is not our concern here. In this paper we examine the relationship between initial conditions for which the energy is placed primarily in a single mode $\gamma$, which we call "mechanical" initial conditions, with initial condition for which the energy is placed in a finite $\delta \gamma$ package of neighboring modes with $\delta \gamma \propto N$, which we call "physical" initial conditions. We find, numerically, that there is a transient time $t \propto \sqrt{N}$, with mechanical initial conditions, which does not exist or is of minor importance for the physical initial conditions. This transient behaviour manifests itself at relatively small $N$ (relatively large $E / N$ ), explored in [2] and [3] but tends to disappear for large $N$, thus preserving the thermodynamic limit. 
The FPU- $\beta$ model is a chain of $N$ masses, coupled to nearest neighbors by hard quartic nonlinear springs. The Hamiltonian representing the chain is

$$
H=\sum_{i=0}^{N} \frac{p_{i}^{2}}{2}+\frac{1}{2}\left(q_{i+1}-q_{i}\right)^{2}+\frac{\beta}{4}\left(q_{i+1}-q_{i}\right)^{4} .
$$

We consider the case of strong springs $(\beta>0)$ and fixed boundaries $q_{0}=q_{N+1}=0$. The constant $\beta$ describing the strength of the anharmonic potential can be scaled to any positive value. We vary the energy and fix $\beta$ at the value 0.1 to compare with previous studies of the FPU lattice. The equations of motion are integrated using a fourth order symplectic integrator. The harmonic part of the Hamiltonian can be put in the form of $N$ independent normal modes via the canonical transformation

$$
q_{i}=\sum_{j=1}^{N} u_{i j} Q_{j} \quad j=1, N
$$

with canonical variables $Q_{j}$. The columns of the matrix $u_{i j}$ are the orthonormal eigenvectors of the positive definite Hermitian eigenvalue problem for the $Q$ 's. The frequencies $\omega_{j}$ of the normal modes $Q_{j}$ are

$$
\omega_{j}=2 \sin \left(\frac{\pi j}{2 N+2}\right)
$$

The above transformation puts the Hamiltonian (1) into the form

$$
H=\sum_{i=1}^{N}\left(\frac{1}{2} P_{i}^{2}+\frac{\omega_{i}^{2}}{2} Q_{i}^{2}\right)+\frac{\beta}{(8 N+8)} \sum_{i, j, k . \ell} \omega_{i} \omega_{j} \omega_{k} \omega_{l} G(i, j, k, \ell) Q_{i} Q_{j} Q_{k} Q_{\ell}
$$

where the coefficients $G$, as calculated in [1], are

$$
G(i, j, k, \ell)=\sum_{P} B(i+j+k+l)
$$

where $P$ represents the eight permutations of sign of $j, k$ and $l$ and the function $B(x)$ takes the value 1 if the argument is zero, -1 if the argument is $\pm 2(N+1)$, and zero otherwise. 


\section{NUMERICAL CALCULATIONS}

The main numerical tool we use is the calculation of the effective number of modes $n_{\text {eff }}$ containing the energy. We use the general formalism of our previous work $[1,2]$ that

the linear energies $E_{i} \equiv \frac{1}{2}\left(P_{i}^{2}+\omega_{i}^{2} Q_{i}^{2}\right), i=1, \ldots, N$, are calculated as a function of time. The information entropy is given by $S=-\sum_{i=1}^{N} e_{i} \ln e_{i}$, where $e_{i}=E_{i} / \sum_{i=1}^{N} E_{i}$ are the normalized energies. We define the effective number of modes sharing the energy by $n_{\text {eff }}(t) \equiv \exp S[1-3]$. We divide $n_{\text {eff }}$ by $N$, to get a fraction in the range from zero to one, which we plot versus time for various values of $N$ and energy densities $E / N$. We also average over 10-20 different realizations of the initial mode phases to minimize fluctuations. We take care to distribute the phases of the modes in a random way so that the quartic term in (1) does not make the total energy very different from the linear energy. In this way one is always close to a set of slightly perturbed linear oscillators as long as $(\beta E / N) \lesssim 1$.

For "physical" initial conditions we distribute the total energy $E$ uniformly among $\delta \gamma=N / 16$ low-frequency modes ranging from $N / 64$ to $5 N / 64$ with randomly chosen phases, for a fixed value of $E / N$ over a range of $N$, and plot $n_{e f f} / N$ versus time. We compare the results to "mechanical" initial conditions in which energy is placed in a fixed number of modes where $\delta \gamma=5$ with the modes ranging from $N / 64$ to $(N / 64)+4$. We have worked with other values of $\delta \gamma$, finding the same qualitative results.

In Figure 1 we show the evolution of $n_{\text {eff }} / N$ at fixed energy density $E / N=0.1$ for $N$ ranging from 256 to 4096 for $\delta \gamma=N / 16$. The data are seen to lie essentially on a universal evolution curve with the correspondence improving for large values of $N$ (error bars, where shown, refer to the statistical error computed over the different initial phases; otherwise errors are of the size of the symbols). This result is verified numerically for energy densities in the range of $E / N[0.03,0.8]$. In Figure 2 we show the evolution of $n_{\text {eff }} / N$ for 
the initial $E / N=0.05$ and for the same range of $N$, as in Figure 1 , but for $\delta \gamma=5$ and centered around $\gamma=(N / 64)+2$. We see that there is an initial transient with the evolution of $n_{\text {eff }} / N$ versus time, different for each $N$-value, but coalescing later in the evolution at longer times and higher values of $n_{e f f} / N$. If we introduce a factor of $\sqrt{N}$ to normalize the time scale (not shown), we then find that the evolution curves coalesce at early times but then diverge. These results are qualitatively consistent with the numerical study in [2] (Fig. 5), which indicated an extra volume filling factor, proportional to $\sqrt{N}$, when the energy was placed primarly in a single low frequency mode, typically $\gamma=3$. In this case the primary driving frequency is a beat $\Omega_{B} \propto \gamma E / N^{2}$, with $\gamma=3$, such that the time for transferring energy is much longer than in the present situation. This allows the filling of the low frequency modes by successive excitations (see ref. [1]) to manifest itself in the additional $\sqrt{N}$ dependence. In Figure 3 we plot $n_{e f f} / N$ as a function of the logarithm of time, for a range of $E / N$ values again with "physical" initial conditions (note that $n_{e f f} / N$ asymptotically converges to a value which is smaller than 1 , due to fluctuations as computed in Ref. [6]). The evolution is a monotonically increasing function with an initial transient and later an approximately linear increase on the logarithmic time scale. The linear part shifts to the left by somewhat less than a decade with every doubling of the energy density, which indicates a power law increase of the time scale with $(N / E)^{\alpha}$ having an approximate exponent $\alpha \simeq 3$. To be more quantitative we will normalize the time by a function of $N / E$, as in [2] (Fig. 5), to determine if all values of $N / E$ can be fitted on a universal curve, after estimating the value of $\alpha$ analytically.

\section{A SCALING ESTIMATE AND NUMERICAL COMPARISON}

In the following we present an approximate theory of Hamiltonian diffusion to explain, qualitatively, the power law behavior at low energy densities. We start by assuming that 
there is an effective number of low-frequency modes $\delta k$ that are responsible for stochastically transferring energy to the high-frequency modes, leading to equipartition. We assume that the initial mode package containing the energy, $\delta \gamma$, is such that $\delta \gamma \leq \delta k$, where the size of the effective package $\delta k$ determines the couplings to high frequency. Because, for early times, most of the energy is in the low-frequency modes, it is convenient to classify the quartic monomial appearing in the sum of (4) depending on how many of the four Q's in it belong to low-frequency modes. The largest quartic terms at early times have the four Q's of low-frequency modes. These couplings produce deformations of the linear actions of the low frequency modes, creating stochastic separatrices for those modes. In [1] it was shown that the necessary energy to create separatrices in the low frequency modes has $E \propto 1 / N$ if energy is placed in a single mode. If we place energy in $\delta \gamma \propto N$ modes we expect stochastic separatrices to be created for $E / \delta \gamma \propto 1 / N$, such that $E$ is independent of $N$. However, this occurs at low energy for $\delta \gamma / N$ small.

Because of the nonlinear couplings among the low-frequency modes, the frequency of these modes is corrected by a beat that we approximate in the following way. We substitute canonical action angle variables $Q_{i}=\sqrt{\left(2 I_{i} / \omega_{i}\right)} \cos \left(\phi_{i}\right)$ and $P_{i}=\sqrt{\left(2 \omega_{i} I_{i}\right)} \sin \left(\phi_{i}\right)$ into the Hamiltonian (4), to obtain

$$
H=\sum_{i} \omega_{i} I_{i}+\left(\frac{\beta}{2 N+2}\right) \sum_{i, j, k, l} G(i, j, k, l) \sqrt{\omega_{i} \omega_{j} \omega_{k} \omega_{l} I_{i} I_{j} I_{k} I_{l}} \operatorname{ang}(i j k l),
$$

where $\operatorname{ang}(i j k l) \equiv \cos \left(\phi_{i}\right) \cos \left(\phi_{j}\right) \cos \left(\phi_{k}\right) \cos \left(\phi_{l}\right)$. The frequency of mode $i$ is the derivative of the Hamiltonian respect to $I_{i}$, which evaluates to

$$
\Omega_{i}=\omega_{i}+\left(\frac{\beta}{N+1}\right) \sum_{j, k, l} G(i, j, k, l) \sqrt{\omega_{i} \omega_{j} \omega_{k} \omega_{l} I_{j} I_{k} I_{l}} \text { ang }(i j k l) / \sqrt{I_{i}} .
$$

We further assume that there is a rapid spreading over low frequency modes, as observed numerically [1], such that we are considering the sum to run over some $\delta k$ modes, to be 
determined. After using the selection rule (5), the number of terms in the above sum is then of the order of $(\delta k)^{2}$. We also assume every quartic term in this sum is typically of the same size, i.e. with equal energies for all low frequency modes, $\omega_{i} I_{i}=E / \delta k$. Since these terms come with random phases, according to standard gaussian statistics we take the sum to be proportional to the square root of the number of terms. With these assumptions, and setting $\omega_{i}=\omega_{j}$ so that $\left(I_{j} / I_{i}\right)^{1 / 2}$ cancels, (7) becomes

$$
\Omega_{i} \approx \omega_{i}+\omega_{i} \frac{\beta E}{N}
$$

with the $\delta k^{-1}$ in the energy per mode cancelling the $\delta k$ effective couplings. In (8) and below we set $N+1 \simeq N$ (large $\mathrm{N}$ ) except where it appears in the selection rule. ¿From (3) $\omega_{i} \simeq \pi i / N$, for low frequency modes, and taking the beat frequency $\Omega_{B}$, between $\omega_{i}$ and a neighboring mode, to be of the order of the nonlinear frequency shift, we obtain, for any $i$ (with $i \ll N$ ),

$$
\Omega_{B} \sim \frac{\pi i}{N} \frac{\beta E}{N}
$$

The change in the linear energy $E_{i}=\frac{1}{2}\left(P_{i}^{2}+\omega_{i}^{2} Q_{i}^{2}\right)$ of a driving low frequency mode $i$, can be calculated by taking the derivative of (6) with respect to the angle $\phi_{i}$

$$
\frac{d E_{i}}{d t}=-\left(\frac{2 \beta}{N+1}\right) \omega_{i} \sum_{j, h 1, h 2} G(i, j, h 1, h 2) \sqrt{\omega_{i} \omega_{j} \omega_{h 1} \omega_{h 2} I_{i} I_{j} I_{h 1} I_{h 2}} \sin \phi_{i} \operatorname{ang}(j, h 1, h 2),
$$

As in our previous work [1] the notation $h 1$ and $h 2$ explicitly indicates that the energy transfer occurs only between low frequency beat oscillation and high frequency mode difference oscillations through the Arnold diffusion mechanism. In the above equation, the summation is over indices $j, h 1$ and $h 2$ for a given $i$. The only terms to transfer energy to high frequency modes are the ones where $j=i$, since then the product of the two low frequency angles does not have a fast phase associated with it. Additionally, the selection 
rule requires that $G(i, j, h 1, h 2)$ will be zero unless

$$
2 i+h 1+h 2=2 N+2,
$$

for which $G=-1$. Expanding the dispersion (3) at high frequency and using (11),

$$
\delta \omega_{h} \simeq\left(\pi^{2} i / 2 N^{2}\right)(h 1-h 2)
$$

In order for the low frequency beat oscillations to transfer energy by Arnold diffusion to the high frequency beats we require $\Omega_{B} \gtrsim \delta \omega_{h}$. From (9) and (12) the inequality gives

$$
\frac{\pi}{N} i \frac{\beta E}{N} \gtrsim \frac{\pi^{2}}{2 N^{2}} i(h 1-h 2)_{\mathrm{Max}}
$$

which reduces to

$$
\delta h \equiv(h 1-h 2)_{\mathrm{Max}} \lesssim \frac{2 \beta E}{\pi}
$$

To determine the number of terms in the sum in (10) we note that for every $i$ we can take $h 2$ arbitrarily from the high frequency package of $\delta h$ modes and then $h 1$ is calculated from (11) with the restriction, from (13) that $h 1-h 2 \lesssim 2 \beta E / \pi$. Writing (11) in the form

$$
i=N+1-h_{2}-\ell / 2 \quad, \quad \ell \text { integer }
$$

such that for $i=1$, we have

$$
\begin{array}{ll}
h_{2}=N-1, & \ell=2 \\
h_{2}=N-2, & \ell=4
\end{array}
$$

up to $i=\delta h$ for which

$$
h_{2}=N-\delta h / 2, \quad \ell=\delta h .
$$

Thus we have a decreasing number of couplings with increasing $i$, with the average number of couplings per low frequency mode $\delta h / 4$ which scales with $\beta E$ as in (13). Substituting 
this result, together with (13), into (10), then for a single low frequency mode $i$ we obtain an estimate for its averaged energy decay

$$
\frac{d E_{i}}{d t}=-\left(\frac{2 \beta}{N}\right) \omega_{i} \frac{\beta E}{2 \pi} E_{i} E_{h}(t)
$$

where from $(3) \omega_{i}=\pi i / N$.

Since $\delta k$ low frequency modes, assumed to have energy, couple to $\delta h$ high frequency modes with $\delta k=\delta h$, the cross-couplings imply each high frequency mode is coupled on average to $\delta k / 4$ low frequency modes. There are phases in the low frequency mode beat oscillations and in the high frequency difference oscillations that can affect the Arnold diffusion. This has only been studied for exponentially slow diffusion [12]. The effect of these phases when more than one driving term exists, for the case of strong Arnold diffusion, $\Omega_{B} \lesssim \delta \omega_{h}$, has not been studied. For lack of evidence we will use the simplest assumption that the effect from each low frequency mode is independent. Setting $\omega_{i}=\beta E / N(i=$ $\delta h / 2=\beta E / \pi)$ as an average value in (14), and dividing by $E_{i}$, we obtain, an average, for each mode in the package

$$
\frac{d E_{i}}{E_{i}}=-\frac{\beta}{\pi}\left(\frac{\beta E}{N}\right)^{2} E_{h}(t) d t,
$$

with the assumption for scaling purposes that the number of couplings is fixed. Integrating (15) in time, with $E_{i}(t)$ varying from $E / \delta k$ at $t=0$ to the equipartition value $E / N$ at the final time $T$ we get

$$
\ln \left(\frac{N}{\delta k}\right) \simeq\left(\frac{\beta}{\pi}\right)\left(\frac{\beta E}{N}\right)^{2} \int_{0}^{T} E_{h}\left(t^{\prime}\right) d t^{\prime}
$$

Equation (15) only holds, initially, since $E$ decreases in time as the diffusion proceeds. However, the change in $E$ is slow compared to the initial build-up of the energy in the high frequency modes. Furthermore, we expect that as the energy in the high frequency modes increases, other pathways become available for the energy distribution among the 
modes, to further justify holding the number of couplings constant in the integration. The final step in the approximation is to estimate the value of $\int_{0}^{T} E_{h}\left(t^{\prime}\right) d t^{\prime}$ at $t=T$, a time of "near-equipartition". The quantity $E_{h}(t)$ appears in an integral, so that its exact form is not required. For a diffusive process, in which the amplitudes of the modes increase with $t^{1 / 2}$, we might expect the mode energies to increase linearly with $t, E_{h}(t) \simeq \frac{t}{T}(E / N)$, such that the time dependence does not depend on $N$. This is found to be approximately true, numerically, over most of the evolution to near-equipartition. Other forms of the time dependence of $E_{h}$ can also be taken with only small numerical differences. Evaluating the integral with the assumption of linear time dependence of $E_{h}(t)$ we obtain

$$
T \simeq \frac{2 \pi}{(\beta E / N)^{3}} \ln \left(\frac{\pi}{2 \beta E / N}\right)
$$

We note that the logarithmic factor varies slowly. The numerical coefficient is only a rough estimate. Equation (11) exhibits a basic scaling of $T \propto(N / E)^{3}$. The scaling can be checked numerically, by rescaling the time in Fig. 3. This is done in Fig. 4, for five values of $E / N$, giving reasonable confirmation of the rescaling of time with $(N / E)^{3}$. We also confirm this scaling by plotting the time to reach $n_{e f f} / N=0.4$ against $E / N$, for all of the data, comparing the result to the inverse cubic scaling (dotted line), in Fig. 5. We can also compare the magnitude of $T$ in (17) with the numerics. Extrapolating the linear (with log time) portion of the $E / N=0.1$ curve in Fig. 3 to $n_{\text {eff }} / N=1$ we obtain, approximately, $T \sim 10^{7}$. Considering our many approximations, this value is remarkably close to the value of $T \simeq 3 \cdot 10^{7}$ obtained from (17).

\section{CONCLUSIONS AND DISCUSSION}

We have indicated, numerically, and justified, theoretically, that the FPU- $\beta$ model has an appropriate thermodynamic limit. Provided there is sufficient energy in a group of low frequency modes that stochastic diffusion to high energy modes occurs on a non- 
exponentially-slow time scale [1], then the dominant time scale to equipartition is a power law $(N / E)^{\alpha}$. The value of $\alpha=3$, estimated from a theoretical scaling argument, was found to fit well to the numerical data.

The numerical results also clarify a result from a previous paper [2] in which a $N^{1 / 2}$ scaling was numerically found, which would not allow a finite-time thermodynamic limit. The resolution of the seeming contradiction is that there is an initial transient which can extend over much of the time to equipartition if $N$ is not very large and the initial energy is placed in the first few modes (not proportional to $N$ ). The existence of a thermodynamic limit also agrees with [4], in which the energy was also placed in a mode packet $\delta \gamma \propto N$. The power of $N / E$, in that study, numerically fit better to $\alpha \simeq 1$. The use of a different equipartition parameter, less sensitive than $n_{e f f} / N$, could have led to uncertainty in $\alpha$, but the issue has not been resolved.

We emphasize that the theory we have developed to explain the scaling, does not predict the shape of $n_{e f f}(t)$ which depends on complicated dynamical processes. Furthermore, $n_{e f f}$ is related to the evolution of the energy in the individual modes in a very complicated way. These dynamics lie beyond a simple mode-averaged theory. We also emphasize that the theory depends on having non-exponentially slow stochastic diffusion to high-frequency modes, being driven by local mode-mixing stochasticity among low-frequency modes [1]. For the approximations to be valid we require that $T \gg \tau$ where $\tau$ is the time scale for the assumed stochastic process. Since $\tau \sim \Omega_{B}^{-1} \sim\left(N^{2} / \beta E\right)^{2}$, the approximations hold if $\beta E / N \ll 1$ 


\section{ACKNOWLEDGMENTS}

We want to acknowledge the support from Fapesp, Brazil, (J.DeL.), NSF Physics, USA, (A.J.L.), and INFN, Italy, (S.R.). 


\section{REFERENCES}

[1] J. De Luca, A. J. Lichtenberg, and M. A. Lieberman, Chaos, 5, 283 (1995).

[2] J. De Luca, A. J. Lichtenberg, and S. Ruffo, Phys. Rev. E, 51, 2877 (1995).

[3] J. De Luca, A. J. Lichtenberg, and S. Ruffo, Phys. Rev. E, 54, 2329 (1996).

[4] H. Kantz, R. Livi, and S. Ruffo, J. Stat. Phys., 76, 627 (1994).

[5] M. Pettini and M. Landolfi, Phys. Rev. A 41, 768 (1990).

[6] C. G. Goedde, A. J. Lichtenberg and M. A. Lieberman, Physica D, 59, 2000 (1992).

[7] G. Tsaur and J. Wang, Phys. Rev. E, 54, 4657 (1996).

[8] L. Casetti, M. Cerruti-Sola, M. Pettini, E. G. D. Cohen, Phys. Rev. E, 55, 6566 (1997).

[9] G. Parisi, Europhys. Lett., 40, 357 (1997).

[10] S. Lepri, Phys. Rev. E, 58, 7165 (1998).

[11] D.L. Shepelyansky, Nonlinearity, 10, 1331 (1997)

[12] B. P. Wood, A. J. Lichtenberg, and M. A. Lieberman, Physica D, 71, 132 (1994). 


\section{FIGURES}

FIG. 1. $n_{e f f} / N$ vs time with $E / N=0.1$ and $\delta \gamma=N / 16$, for $N=256,512,1024,2048$ and 4096 . The error bars are the rms variation over (typically) 10 independent trials, which are averaged to give final values.

FIG. 2. $n_{e f f} / N$ vs time with $E / N=0.05$ and $\delta \gamma=4$ for $N=256,512,1027,2048$. Error bars as in Fig. 1.

FIG. 3. $n_{e f f} / N$ vs time for $N=2048$ and $E / N=0.03,0.05,0.1,0.2,0.4$, and 0.8 .

FIG. 4. $n_{e f f} / N$ vs $t(\beta E / N)^{3}$.

FIG. 5. $t\left(n_{e f f} / N=0.4\right)$ vs $E / N$ compared to proportionality $t$ vs $(E / N)^{3}$ of dashed line. 


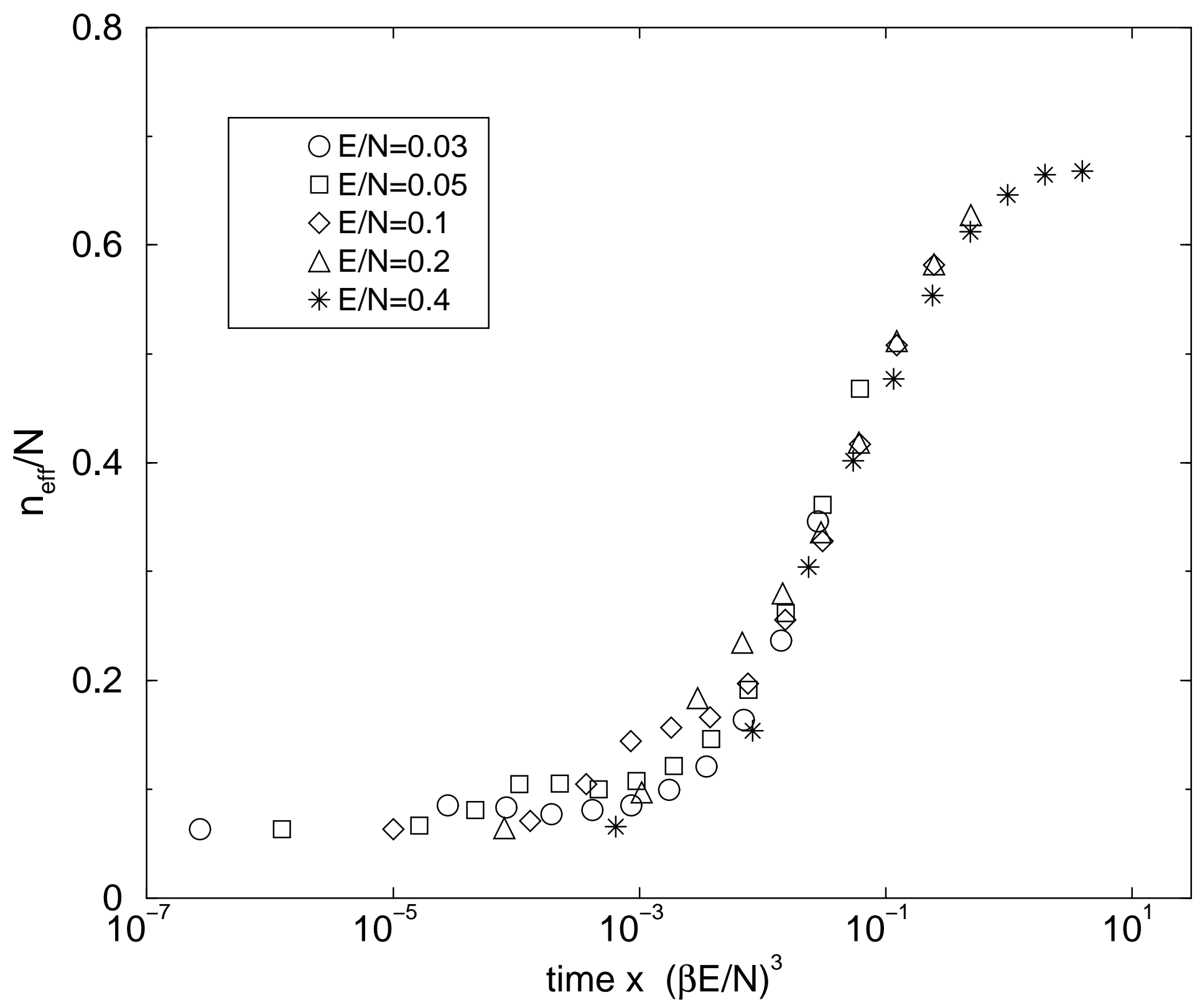




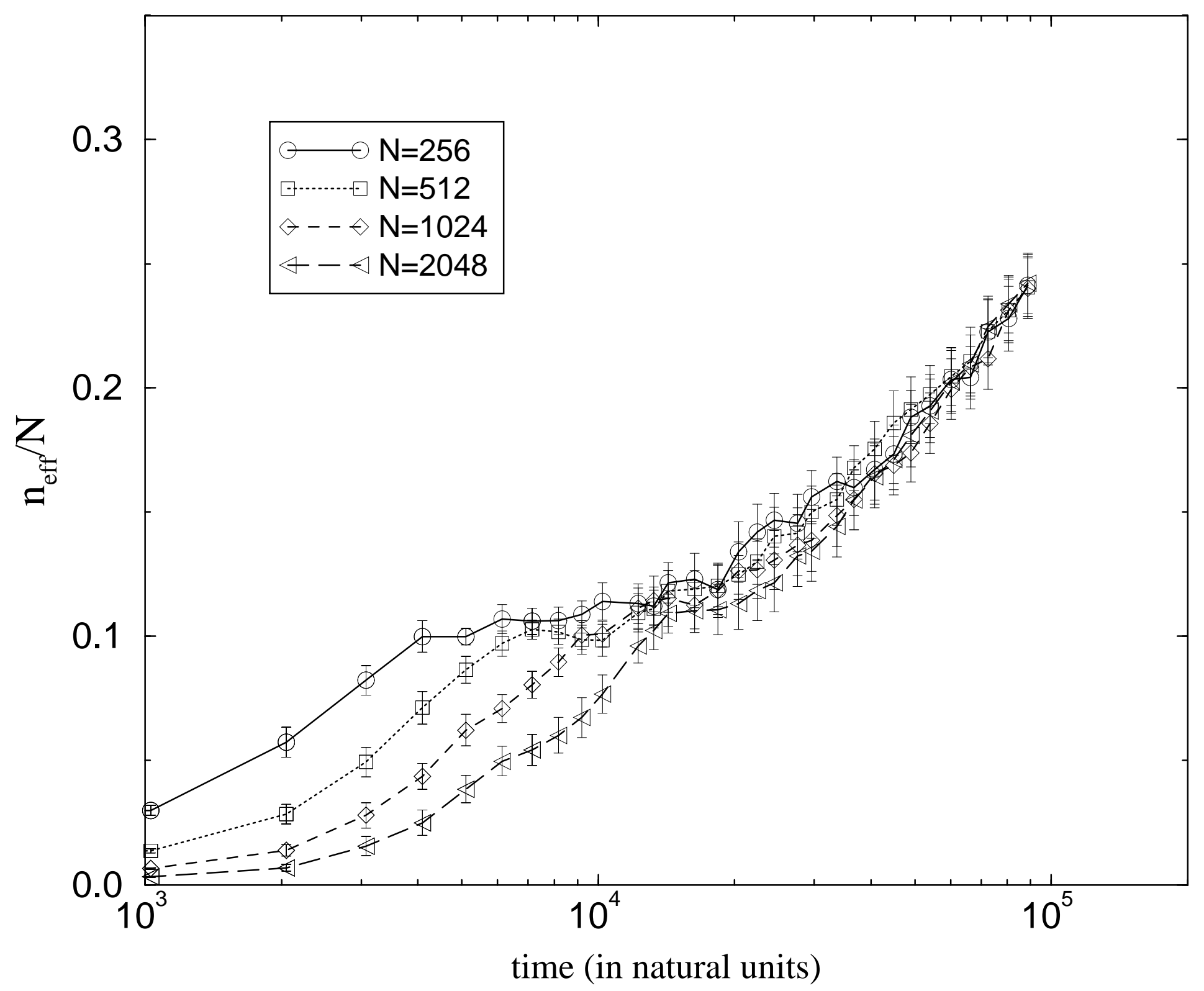




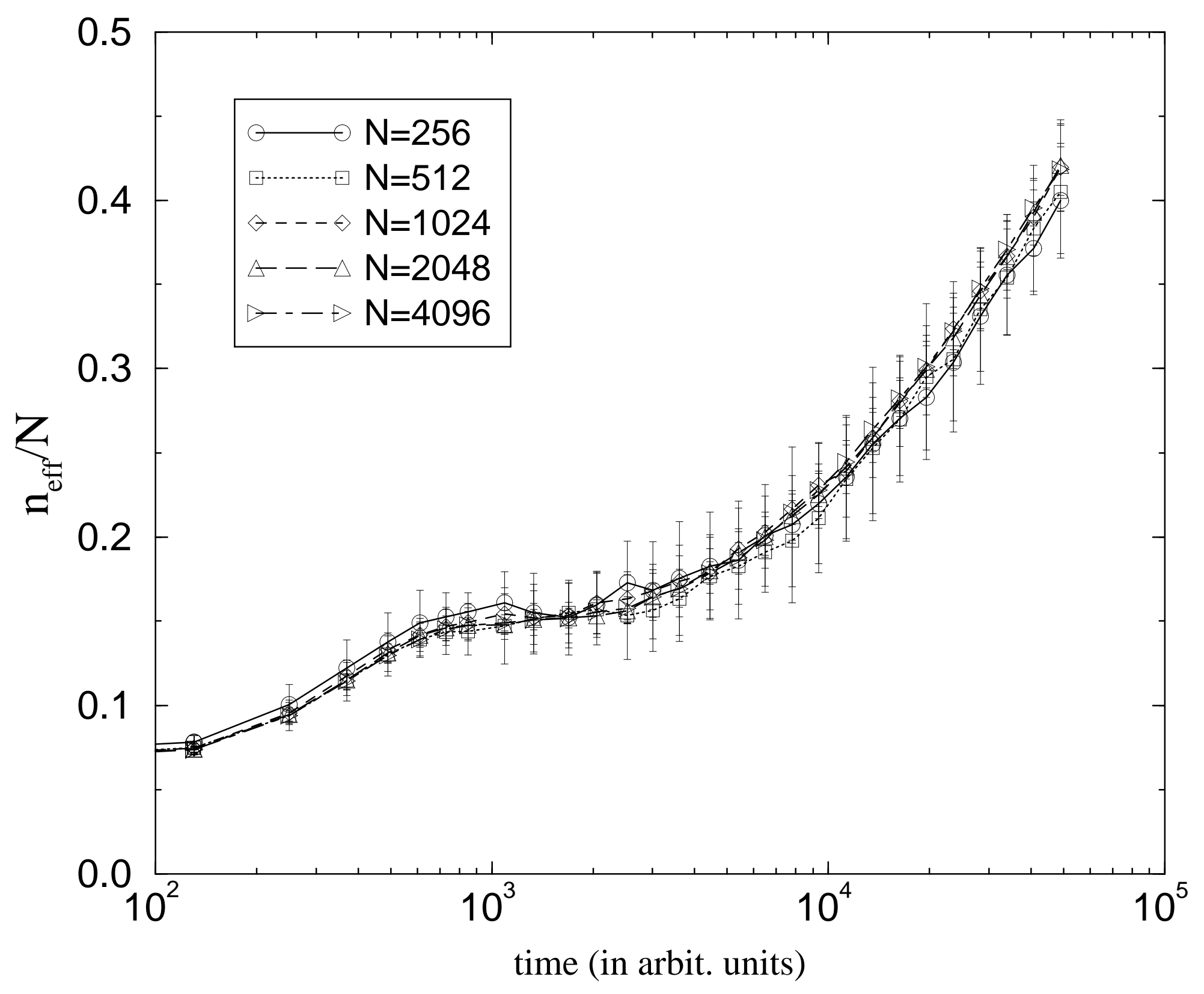




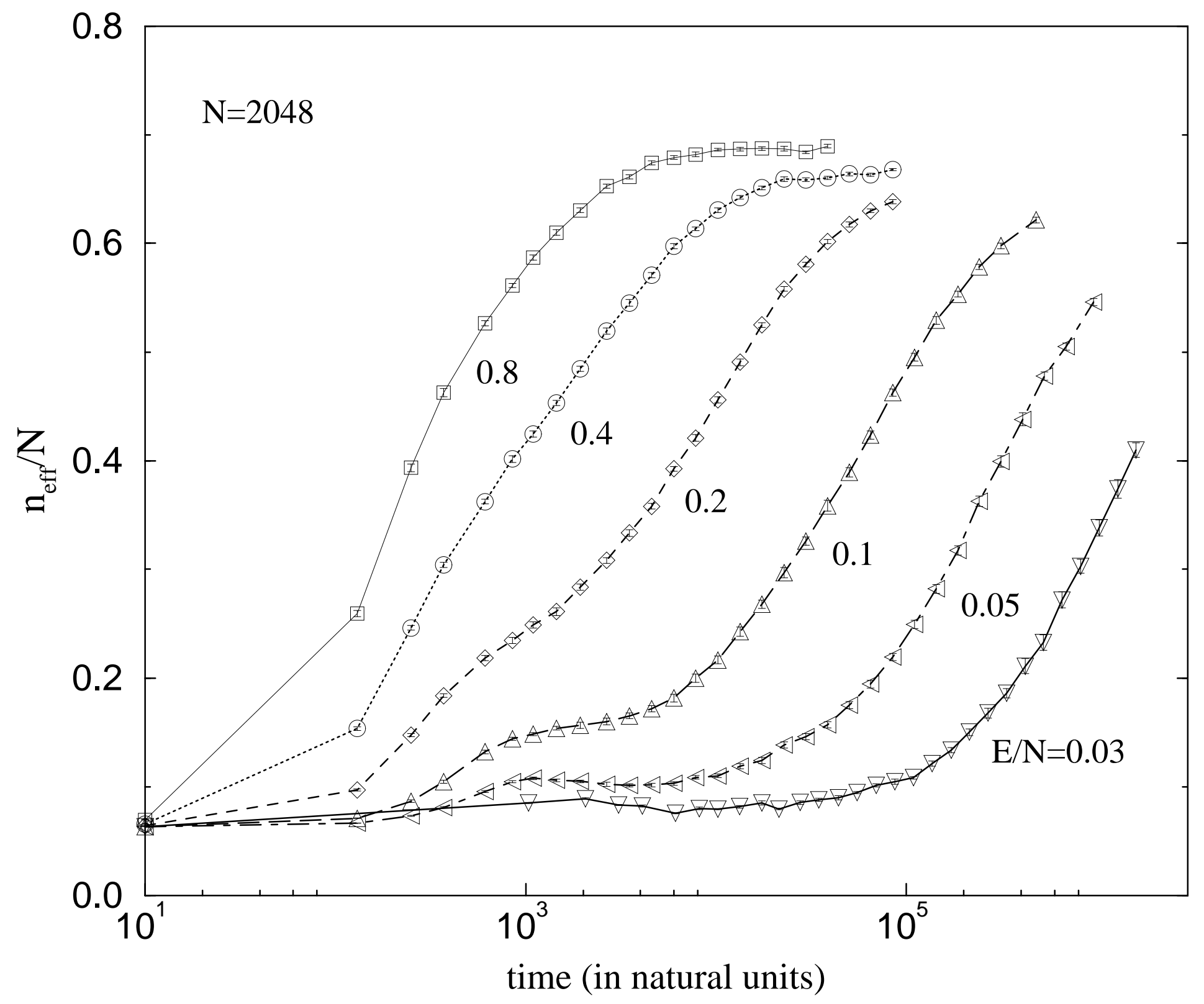




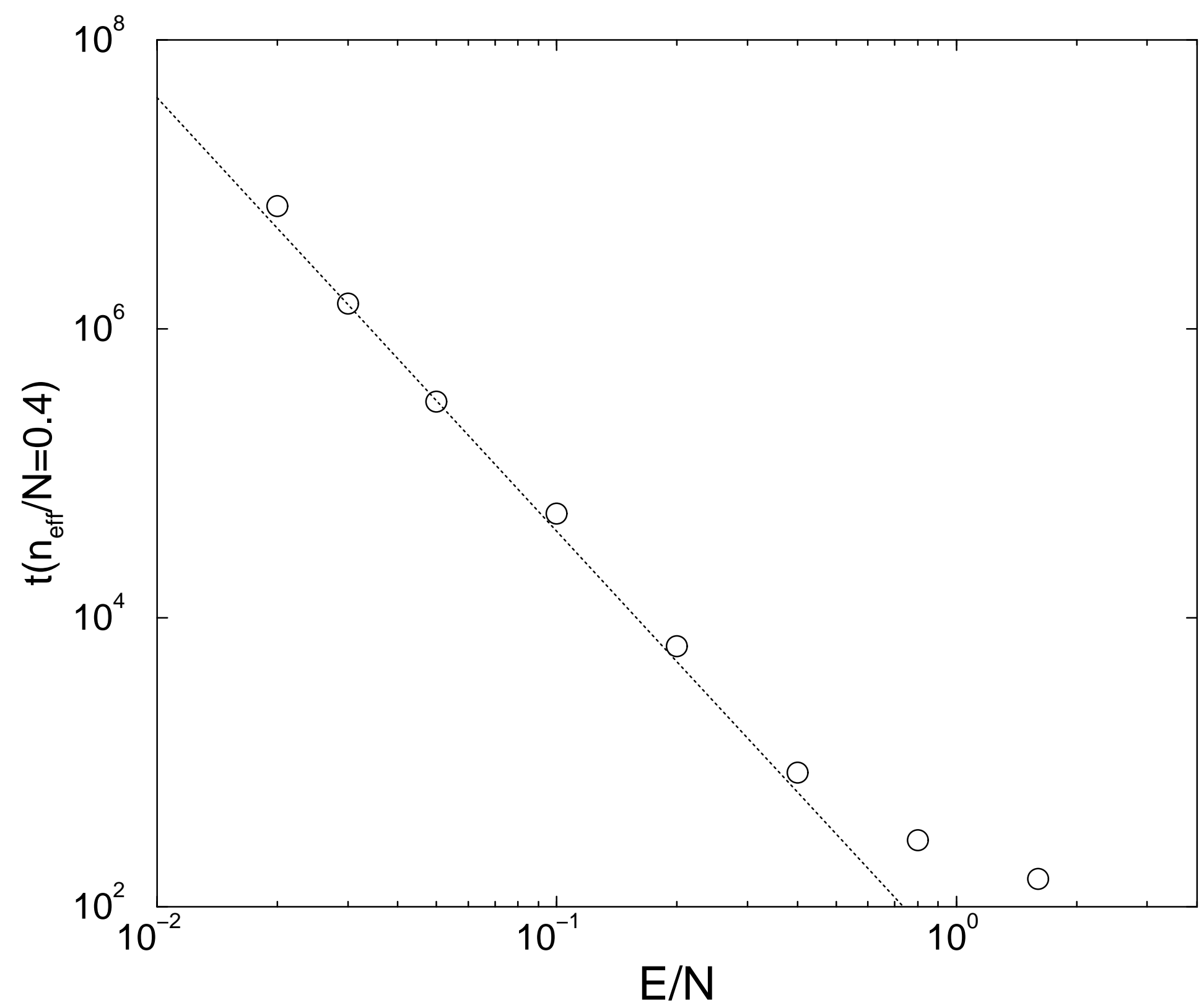

